\title{
Application of Uniform Design Genetic Algorithm in Separation Condition Optimization of 14 Drugs
}

\author{
T. Zhang, X.G. Chen \\ Department of Forensic Chemistry \\ National Police University of China \\ Liaoning Shenyang 110854
}

\begin{abstract}
An optimization method based on uniform design in conjunction with genetic algorithm was developed and applied in the optimization of separation condition of 14 drugs in reversedphase ion pair chromatography and satisfactory optimal result was obtained.
\end{abstract}

\section{Keywords--genetic algorithm; liquid chromatography; condition optimization}

\section{INTRODUCTION}

With the development of theory, technique and instruments, high performance liquid chromatography has become the most efficient separation tool in the analysis of complex samples such as environmental samples, biological samples, medical samples and medical quality control [1]. The complexities of real samples have showed the necessity of optimization of chromatographic separation conditions [2].

Genetic algorithm is an optimization algorithm that mimics the mechanisms of natural selection described by genetics and the Darwinian theory of evolution [3]. As global searching algorithm, genetic algorithm makes use of artificial intelligence to obtain the solution of rather complex problems, so it has been widely applied in resolving combinatorial optimization problems [4], and attempter problems [5] due to their parallelism and effective utilization of global information.

In this article, according to traditional optimization methods, a new strategy based on uniform design and genetic algorithm was established. The optimization strategy was described in details and a software package was developed. The optimization strategy was applied in the optimization of separation condition of 14 drugs in reversed-phase ion pair chromatography and satisfactory optimal result was obtained.

\section{OPTIMIZATION STRATEGY}

The outline of the optimizing process based on genetic algorithm is shown in Figure 1. The procedures including selection of column system, screening for mobile phase and components, searching for the optimization point of mobile phase, has been discussed in our past work [6].

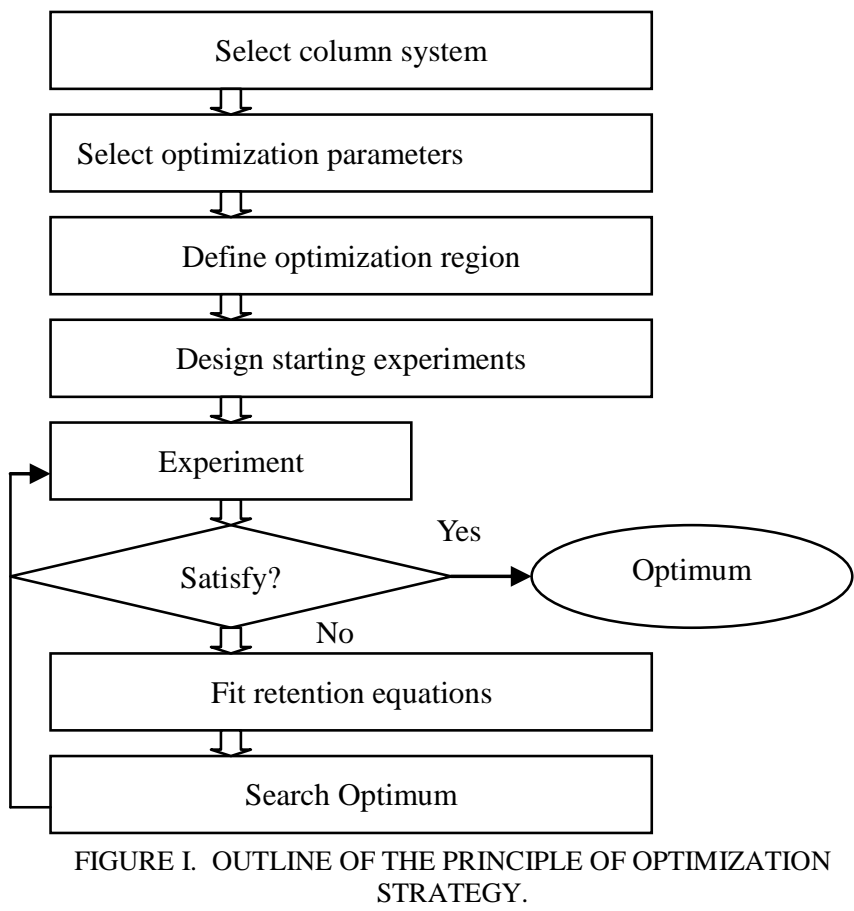

\section{EXPERIMENTAL CONDITIONS}

A. Apparatus

Waters HPLC 515 pump and 996 Photodiode Array Detector (Waters, USA); the separations were carried out on an ODS column $(250 \times 4.6 \mathrm{~mm}$ i.d., $5 \mu \mathrm{m})$; mobile phase were methanol-0.10\% triethylamine (adjusted $\mathrm{pH}$ as 5.50 with acetic acid, $10.0 \mathrm{mmol} / \mathrm{L}$ sodium 1-heptanesulfonate) (49: 51); flow rate was $1.0 \mathrm{~mL} / \mathrm{min}$; detection wavelength was set as $212 \mathrm{~nm}$ and the injection volume was $10 \mu \mathrm{L}$.

\section{B. Reagent}

All drugs including caffeine, morphine, monoacetyl morphine, codeine, strychnine, ephedrine, thebaine, heroin, acetyldihydrocodeine, ketamine, tramadol, papaverine, pethidine, narcotin were purchased from the National Institute for the Control of Pharmaceutical and Biological Products (Beijing, China); methanol, triethylamine, acetic acid and sodium 1-heptanesulfonate were all of RP grade and purchased from Shenyang Regent Factory (Shenyang, China). 


\section{RESULTS AND DISCUSSION}

According to reports in literatures [7] and our past work [8], in this experiment reversed-phase ion pair highperformance liquid chromatography with ODS column was chosen as the separation mode for 14 drugs. The mobile phase was composed of methanol- $0.10 \%$ triethylamine buffer at $\mathrm{pH}$ 5.50 (adjusted using acetic acid) and sodium 1heptanesulfonate was chosen as ion pair reagent. The volume fraction of methanol and the concentration of sodium 1heptanesulfonate were selected the optimization variables ranging from 10 to $90 \%$ (v\%) and from 1.0 to $20.0 \mathrm{mmol} / \mathrm{L}$, respectively. The optimum was obtained with the optimization strategy applied uniform design and genetic algorithm according to the principle of the optimization strategy as shown in Figure 2, the concentrations of methanol was $49 \%$ and sodium 1-heptanesulfonate was $10 \mathrm{mmol} / \mathrm{L}$, respectively. The experimental results and predicted data are listed in table 1 and the separation chromatogram of 14 drugs under the optimal experimental conditions is listed in Figure 3, which confirmed that the accuracy to search the optimal condition was very high and the prediction conditions was satisfying.

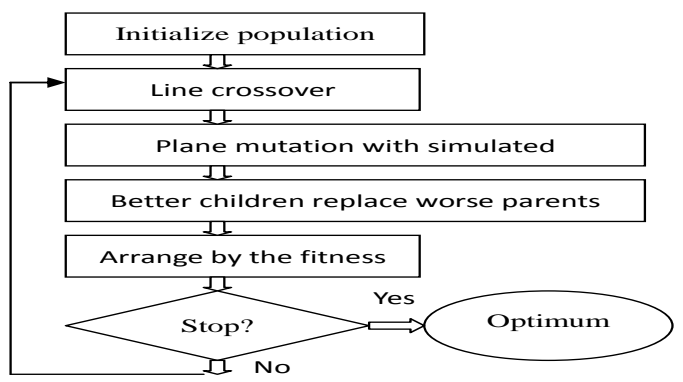

FIGURE II. OUTLINE OF THE PROCESS OF GENETIC ALGORITHM.

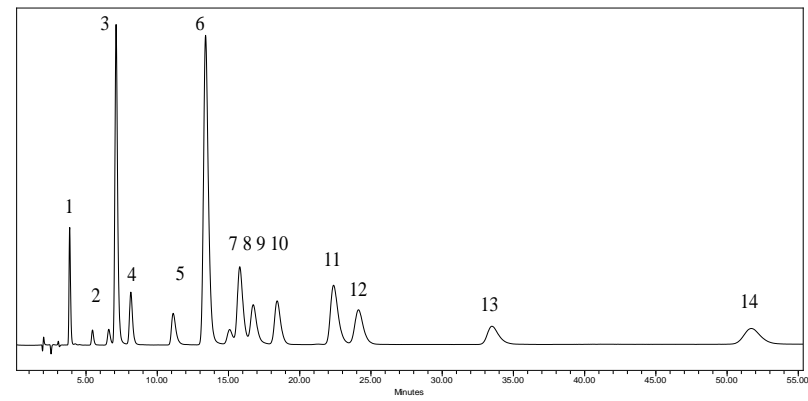

FIGURE III. CHROMATOGRAM OF 14 DRUGS UNDER THE OPTIMAL CONDITIONS.

Furthermore, the optimization method mentioned here has two characteristics. One is uniform design, which makes it possible that the starting experiments are uniformly located and the retention of solutes is more accurately predicted in the range of variables to be optimized. Therefore, the disadvantages of the sequential approaches such as simplex for the local optimum can be overcome. Another is genetic algorithm, which makes it possible to increase the accuracy for prediction of the migration times of solutes. Moreover, the global optimum can be obtained.
TABLE I. PREDICTED (TP) AND EXPERIMENTAL (TE) RETENTION TIME OF 14 DRUGS AT THE OPTIMUMS AND RELATIVE DIFFERENCE (RD) BETWEEN THEM.

\begin{tabular}{ccccc}
\hline Solute & \multirow{2}{*}{ No. } & \multicolumn{3}{c}{ First optimum } \\
\cline { 3 - 5 } & & tp/min & te/min & RD(\%) \\
\hline Caffeine & 1 & 3.98 & 4.06 & 2.01 \\
Morphine & 2 & 5.53 & 5.56 & 0.54 \\
Monoacetyl Morphine & 3 & 7.14 & 7.05 & 1.26 \\
Codeine & 4 & 8.15 & 8.21 & 0.74 \\
Strychnine & 5 & 11.21 & 11.43 & 1.96 \\
Ephedrine & 6 & 13.82 & 13.66 & 1.16 \\
Thebaine & 7 & 15.01 & 14.87 & 0.93 \\
Heroin & 8 & 15.96 & 15.74 & 1.38 \\
Acetyldihydrocodeine & 9 & 16.99 & 17.25 & 1.53 \\
Ketamine & 10 & 18.55 & 18.32 & 1.24 \\
Tramadol & 11 & 22.41 & 22.93 & 2.32 \\
Papaverine & 12 & 24.36 & 25.01 & 2.67 \\
Pethidine & 13 & 33.25 & 33.92 & 2.02 \\
Narcotin & 14 & 51.83 & 50.03 & 3.47 \\
\hline RDmax & & & 3.47 & \\
RDave & & & 1.66 & \\
\hline
\end{tabular}

\section{V.CONCLUSION}

The optimization method using uniform design and genetic algorithm was applied in optimizing the chromatographic separation in reversed-phase ion pair chromatography mode. The process of optimizing both the content of methanol and sodium 1-heptanesulfonate (ion pair reagent) in the mobile phase using uniform design and simulated annealing genetic algorithm was introduced in detail. 14 drugs were selected to test the optimization method and the optimum was obtained by optimization procedures, the mean relative deviation between predicted and experimental values was only $1.66 \%$ under the optimum separation condition. The results shown that the method using uniform design and genetic algorithm is efficient and it has potential application not only in the optimization of two parameters, but also of much more than two parameters.

\section{ACKNOWLEDGMENT}

Financial support from the National Natural Science Foundation of Liaoning Provence (No. 201102246) is gratefully acknowledged.

\section{REFERENCES}

[1] H. F. Zou, Y. K. Zhang, P. Z. Lu. Ion Pair High Performance Liquid Chromatography. Henan: Henan Science and Technology Press, 1994. 108.

[2] E. J. Klein, S. L. Rivera, J. E. Porter. Optimization of ion-exchange protein separations using a vector quantizing neural network. Biotechnology Progress, 2000, 16 (3): 506-512.

[3] W. Y. Li, H. T. Rasmussen. Strategy for developing and optimizing liquid chromatography methods in pharmaceutical development using computer-assisted screening and Plackett-Burman experimental design. Journal of Chromatography A, 2003, 1016 (2): 165-180.

[4] J. F. Li, H. Liao, B. Normand, C. Cordier, G. Maurin, J. Foct, C. Coddet. Uniform design method for optimization of process parameters of 
plasma sprayed TiN coatings. Surface \& Coatings Technology, 2003, $176(1): 1-13$

[5] K.M. Rehana, K.S. Beng. Optimization of the simultaneous batch determinations of $\mathrm{Bi}$ (III), $\mathrm{Hg}$ (II) and $\mathrm{Cu}$ (II) at an epoxy-graphite electrode bulk modified with 2-mercaptobenzothiazole. Analyst, 2001, 126 (12): 2172-2177.

[6] X. G. Chen, X. Li, L. Kong, J. Y. Ni, R. H. Zhao, H. F. Zou. Application of uniform design and genetic algorithm in optimization of reversedphase chromatographic separation. Chemometrics and Intelligent Laboratory Systems, 2003, 67: 157-166.

[7] M. Zecevic, L. Zivanovic, S. Agatonovic-Kustrin, D. Minic. The use of a response surface methodology on HPLC analysis of methyldopa, amiloride and hydrochlorothiazide in tablets. Journal of Pharmaceutical and Biomedical Analysis, 2001, 24 (5-6): 1019-1025.

[8] X. G. Chen, R. H. Zhao, J. Y. Ni, H. F. Zou. Application of simulated annealing genetic algorithm in optimization of linear gradient-elution conditions in high performance liquid chromatography. Chinese Journal of Chromatography, 2004, 22 (1): 87. 